\title{
Efecto de metformina sobre la expresión del factor de necrosis tumoral- $\alpha$, los receptores Toll-like 2/4 y la PCR ultra sensible en sujetos obesos con diabetes tipo 2
}

\section{Effect of metformin on the expression of tumor necrosis factor- $\alpha$, Toll like receptors $2 / 4$ and $C$ reactive protein in obese type- 2 diabetic patients}

Background: The pharmacological action of metformin goes beyond mere glycemic control, decreasing markers of inflammation and contributing to the reduction of oxidative stress. Aim: To evaluate biochemical, anthropometric and pro-inflammatory markers in obese type 2 diabetic patients treated or not with metformin. Patients and Methods: Obese patients with type 2 diabetes were invited to participate in the study if they were aged more than 40 years, were not receiving insulin, did not have cardiovascular diseases and were not taking anti-inflammatory drugs. A pharmacological history was taken and patients were stratified in two groups whether they were using metformin or not. A fasting blood sample was obtained to measure blood glucose, insulin, lipid levels, C reactive protein (hsCRP) and to isolate peripheral blood mononuclear cells. RNA was isolated from these cells to measure expression of tumor necrosis factor- $\alpha$ (TNF- $\alpha$ ), Interleukin-6 (IL-6), nuclear factor kappa-light-chain-enhancer of activated B cells (NF-КB), Toll-Like Receptor 2/4 (TLR 2/4) and beta-2-microglobulin (B2M). Results: Thirty participants were studied. Of these, 16 subjects aged $54.4 \pm 5.5$ years were treated with metformin and 14 subjects aged $54.9 \pm 6.4$ years did not receive the drug. Participants receiving metformin had lower levels of hsCRP and lower $m R N A$ relative abundance of TNF- $\alpha$ and TLR 2/4. There were no differences in glucose levels or lipid profile between both groups. Conclusions: Obese diabetic patients treated with metformin had lower levels of hsCRP expression of TNF- $\alpha$ and TLR 2/4, than their counterparts not receiving the drug.

(Rev Med Chile 2012; 140: 1377-1382).

Key words: Inflammation; Metformin; Tumor Necrosis Factor-alpha; C-reactive protein.

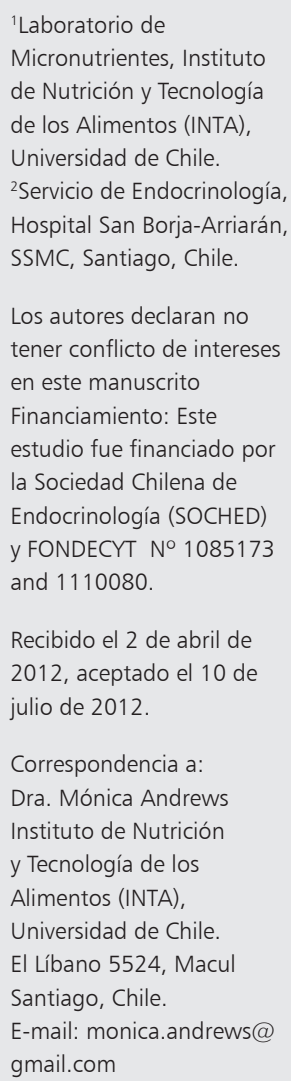

L a diabetes mellitus tipo 2 (DT2) se caracteriza por cursar con hiperglicemia y con un elevado nivel de marcadores inflamatorios circulantes, tales como: proteína C reactiva (PCR), interleuquina (IL)-6, factor de necrosis tumoral (TNF) $\square$ y fibrinógeno ${ }^{1,2}$. Tanto la hiperglicemia como la inflamación constituyen factores de riesgo para el desarrollo de complicaciones de la diabetes, tales como enfermedad cardiovascular y nefropatía ${ }^{3,4}$.

La hiperglicemia por una parte provoca la formación de especies de glicación avanzada (AGEs) y estas especies a su vez promueven el estrés oxidativo con la producción de especies reactivas 
al oxígeno (ROS), los que juegan un rol en la progresión de la diabetes y en las complicaciones de esta enfermedad ${ }^{5}$.

La inflamación en la diabetes tiene distintos orígenes. Por una parte, es promovida por la secreción de citoquinas en el tejido adiposo cuando la diabetes es acompañada por obesidad. El tejido adiposo tiene la función de almacenar eficientemente la energía, pero también funciona como un órgano endocrino, ya que de él se liberan distintas quimoquinas y citoquinas bio-activas, tales como TNF- $\square$, IL-6, IL1, etc, lo que se observa exacerbado cuando este tejido además esta infiltrado por macrófagos $^{6-8}$. La secreción de estas citoquinas provoca un círculo vicioso en la obesidad, ya que en la medida que comienzan a aumentar su concentración en la circulación, se produce la disfunción del tejido adiposo, se reduce la sensibilidad a la insulina del adipocito y con ello aumenta aun más la secreción de citoquinas.

La síntesis de TNF- $\square$ e IL-6 también puede ser estimulada por el aumento de glucosa y de ácidos grasos circulantes, en distintos tejidos además del adiposo, incluyendo, hígado y músculo ${ }^{9,10}$. La glucosa, los ácidos grasos y sus metabolitos resultantes de su oxidación incompleta pueden inducir directamente tanto la expresión del gen como la síntesis de la proteína de ambas citoquinas ${ }^{10}$, pero también lo pueden hacer mediante una vía indirecta que es a través de la activación de los receptores Toll like (TLR) 2/4, la vía actúa mediante el aumento de actividad de la NAPH oxidasa lo que provoca la activación de NF- $\square \mathrm{B}^{11,12}$. La activación de NF- $\square$ B es un evento clave en la amplificación de la inflamación, ya que este factor nuclear regula la expresión de genes de la inflamación.

Metformina es un fármaco perteneciente a la familia de las biguanidas, es un agente insulinosensibilizador y también disminuye la producción de glucosa hepática. Metformina es el fármaco de elección en el tratamiento de la DT2 ${ }^{13}$. En el estudio prospectivo de diabetes del Reino Unido (UKPDS), la metformina fue el único agente que redujo el infarto al miocardio y las muertes asociadas a diabetes ${ }^{14}$. Posteriormente, se observó que el uso de metformina en sujetos diabéticos obesos produjo una reducción del índice de masa corporal (IMC), con una disminución de la grasa tanto periférica como central, lo que se tradujo en una disminución de la resistencia a la insulina y de secreción de citoquinas pro-inflamatorias ${ }^{15,16}$.
Asimismo, en ratas a las que se les indujo diabetes mediante la inyección con estreptozotocina, el tratamiento con metformina redujo el estrés oxidativo y la expresión de TNF- $\mathrm{e}$ e IL- ${ }^{17}$.

El objetivo del presente estudio fue evaluar los parámetros bioquímicos, antropométricos y proinflamatorios mediante la expresión de genes de la inflamación en sujetos con diabetes mellitus tipo 2 obesos tratados con y sin metformina.

\section{Material y Métodos}

\section{Sujetos}

Se estudió 30 sujetos hombres con diabetes mellitus tipo 2 obesos. Los criterios de inclusión para este estudio fueron: ser mayores de 40 años y sin tratamiento con insulina para el control de la diabetes. Entre los criterios de exclusión poseer antecedentes de enfermedades cardiovasculares, 10 o más años de diagnóstico de la enfermedad y tratamiento con anti-inflamatorios. Todos los sujetos eran atendidos en la Unidad de Diabetes del Hospital San Juan de Dios, Santiago. Cada sujeto firmó un consentimiento informado previamente aprobado por el Comité de Bioética del INTA.

\section{Determinaciones antropométricas y bioquímicas}

Los sujetos fueron pesados y medidos para obtener luego el IMC individual, además se determinó la circunferencia abdominal y la presión arterial. Se les realizó un cuestionario para conocer los fármacos que ingerían, con lo que finalmente se conformaron los dos grupos: tratados con y sin metformina. Posteriormente, se obtuvo $30 \mathrm{ml} \mathrm{de}$ sangre por punción venosa después de un ayuno de $8 \mathrm{~h} ; 12 \mathrm{ml}$ fueron utilizados para la determinación de parámetros bioquímicos (glicemia, insulina, perfil lipídico, PCR ultra sensible) y los 18 restantes fueron utilizados para aislar células mononucleares periféricas (CMPs).

\section{Obtención de células mononucleares periféricas (CMPs)}

Las CMPs fueron aisladas mediante gradiente de sedimentación con Ficoll (Histopaque 1077, densidad 1.119. Sigma Diagnostics, St Louis, MO). Las CMPs fueron removidas y lavadas dos veces con PBS y luego centrifugadas a 2.000 g por $5 \mathrm{~min}$ y posteriormente lavadas con PBS. Las alícuotas fueron separadas para la obtención de ARN y almacenadas a $-80^{\circ} \mathrm{C}$. 
Metformina e inflamación en diabetes tipo 2 - M. Andrews et al

\section{PCR en tiempo real}

El ARN de las CMPs fue extraído utilizando trizol (Invitrogen) de acuerdo a las instrucciones del fabricante. Posteriormente, se realizó síntesis de cDNA con $1 \mathrm{mg}$ de ARN utilizando el kit AffinityScript cDNA synthesis (Stratagene). La PCR en tiempo real fue realizada con el Master Mix Brilliant II SYBR Green QPCR (Stratagene) en un equipo Max Proo System 3000. Como genes endógenos de utilizó la gliceraldehído-3fosfato deshidrogenasa (GADPH) y la beta2-microglobulina (B2M). Las secuencias de los partidores usados fueron las siguientes: GADPH: CCAGCAAGAGCACAAGAGGA y TCAAGGGGTCTACATGGCAA; B2M: GATGCCGCATTTGGATTGGA y TGGAGCAACCT-GCTCAGATA; NFपB: TGCATCCAAAGGTGCTCAGA y GCAGCTGGCAAAGCTTAGTA; IL6: ATGTCTGAGGCTCATTCTGC y GCGGCTACATCTTTGGAATC; TNFП: GTTCCTCA-GCCTCTTCTCCT y ACAACATGGGCTACAGGCTT; TLR2: AGATGCCTCCCTCTTACC-CATGTT y AAGACTTTGGCCAGTGCTTGCT; TLR4: AGGAACAGTGGGTACAGGATG-CAA y TCACCCTTAGCATAAGGCCTGACA.

\section{Análisis estadístico}

El análisis estadístico fue llevado a cabo en el software GraphPad Prism 4. Los resultados de las mediciones antropométricas y bioquímicas fueron expresados como promedios $\pm \mathrm{ES}$. Las diferencias estadísticas de los parámetros antropométricos y bioquímicos fueron evaluadas mediante $\mathrm{T}$ de student. Los resultados del PCR en tiempo real están normalizados al gen endógeno y están presentados como veces de cambio, las diferencias fueron analizadas mediante $\mathrm{T}$ de student. Las diferencias se consideraron significativas con un $\mathrm{p}<0,05$.

\section{Resultados}

Se estudiaron 30 sujetos obesos con diabetes tipo 2 sin requerimiento de insulina. De ellos, 16 pacientes utilizaban metformina para el control de la enfermedad, la dosis promedio utilizada por los sujetos era de $878 \pm$ $324 \mathrm{mg} /$ día, de los restantes 14 sujetos, 10 utilizaban glibenclamida y 4 se manejaban sólo con dieta. Todos los pacientes ingerían aspirina a bajas dosis e ingerían algún hipolipemiante, ya sea atorvastatina o lovastatina. Para el tratamiento de la hipertensión arterial los pacientes ingerían enalapril o losartán. Todos los sujetos en estudio eran no fumadores.

Los sujetos de ambos grupos eran comparables en edad, IMC, circunferencia abdominal y glicemia, sin embargo, los individuos que estaban bajo tratamiento farmacológico con metformina para el control de la diabetes tuvieron una menor insulina basal (T Student: $\mathrm{p}<0,05 ; \mathrm{t}=2,77)$ y también una menor PCRus ( $\mathrm{T}$ Student: $\mathrm{p}<0,05 ; \mathrm{t}=1,94)$ (Tabla 1 ). No se observó diferencias en el perfil lipídico de ambos grupos A pesar de los niveles de triglicéridos plasmáticos ninguno de los pacientes estaba bajo tratamiento con fibratos. Los sujetos bajo tratamiento con metformina tenían menor presión arterial sistólica que los pacientes sin este fármaco.

La abundancia relativa de los genes asociados a la inflamación también fue distinta en aquellos que consumían metformina con los que no la consumían. La expresión de TNF- $\square$ (T Student: $\mathrm{p}<0,05 ; \mathrm{t}=2,3$ ) y de los TLR 2/4 (T Student: $\mathrm{p}<0,05 ; \mathrm{t}=1,67 ; \mathrm{p}<0,05, \mathrm{t}=1,35$;

Tabla 1. Características antropométricas y bioquímicas de población estudiada

\begin{tabular}{|lcc|}
\hline & $\begin{array}{c}\text { OBDM } \\
\text { c/metformina } \\
\mathbf{n}=\mathbf{1 6}\end{array}$ & $\begin{array}{c}\text { OBDM } \\
\text { s/metformina } \\
\mathbf{n}=\mathbf{1 4}\end{array}$ \\
\hline Edad (años) & $54,4 \pm 5,5$ & $54,9 \pm 6,4$ \\
\hline Peso (Kg) & $93,0 \pm 8,0$ & $91,8 \pm 5,7$ \\
\hline IMC (Kg/mt $\left.{ }^{2}\right)$ & $32,2 \pm 2,3$ & $31,6 \pm 1,3$ \\
\hline Cir. abdominal (cm) & $106,6 \pm 7,8$ & $106,6 \pm 6,2$ \\
\hline Glicemia basal (mg/dl) & $205,8 \pm 70,7$ & $157,9 \pm 96,2$ \\
\hline Insulina (ng/ml) & $15,9 \pm 8,7$ & $26,0 \pm 10,9$ * \\
\hline PCRus ( $\mathrm{mg} / \mathrm{dl})^{1}$ & $2,7(0,1-5,9)$ & $5,1(0,7-13,6)$ \\
\hline Colesterol total (mg/dl) & $206,0 \pm 50,5$ & $189,5 \pm 53.6$ \\
\hline Col. HDL (mg/dl) & $32,0 \pm 9,3$ & $30,1 \pm 9,4$ \\
\hline Col. LDL (mg/dl) & $119,0 \pm 47,9$ & $131,6 \pm 62,9$ \\
\hline Triglicéridos (mg/dl) ${ }^{1}$ & $261,7(86,7-644,6)$ & $222,9(86,9-968,9)$ \\
\hline
\end{tabular}

IMC: Indice de masa corporal. PCRus: Proteína $C$ reactiva ultrasensible. Los datos son presentados como promedio $\pm \mathrm{ES}$. Prueba $\mathrm{t}$ de student. ${ }^{*} p<0,05$. 'Promedio geométrico, entre paréntesis los rangos. 

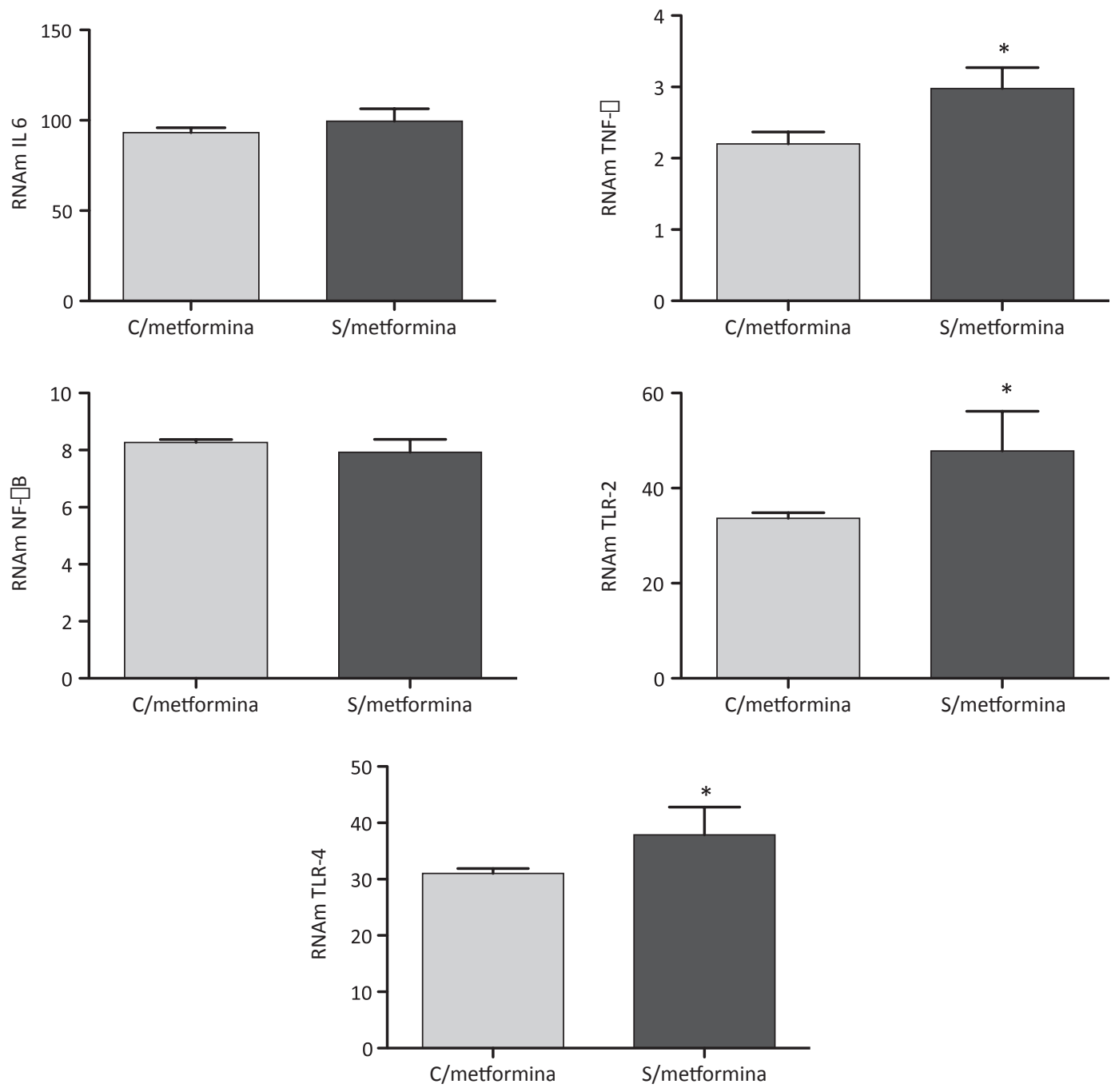

Figura 1. Abundancia relativa de IL-6, TNF-, , NF- $\square$ B, TLR-2 y TLR-4 de sujetos diabéticos tipo 2 obesos con y sin metformina. Los resultados están normalizados a los genes endógenos GADPH y B2M y están expresados como veces de cambio, las diferencias entre grupos fueron analizadas con el test $t$ student. ${ }^{*} p<0,05$.

respectivamente) fue menor en los pacientes con tratamiento con metformina, en cambio la expresión de NF- $\square B$ (T Student: $p>0,05 ; t=0,65$ ) e IL-6 (T Student: $p>0,05 ; t=0,82)$ no fue distinta en ambos grupos (Figura 1).

\section{Discusión}

Una de las metas clásicas a alcanzar en los pacientes diabéticos es el control glicémico, sin embargo, hay estudios que han mostrado que en el desarrollo de las complicaciones de la diabetes hay más factores implicados que sólo la hiperglicemia y la glicación de proteínas ${ }^{18}$. En efecto, el estudio prospectivo UKPDS demostró que el tratamiento con metformina disminuyó la mortalidad y los eventos cardiovasculares comparados con la insulina y las sulfonilureas, aunque tuvieran los mismos efectos en la disminución de la glicemia en una población de diabéticos con sobrepeso ${ }^{14}$. En el presente estudio no encontramos diferencias en la glicemia de sujetos tratados con metformina 
y sin ella, sin embargo, se observó que los sujetos bajo tratamiento con metformina tenían un menor nivel de PCRus circulante.

La PCR es uno de los marcadores de la inflamación mejor caracterizado a la fecha. Elevados niveles de PCR se observan en sujetos con síndrome metabólico y se acepta que la elevación de la PCRus constituye un riesgo para la enfermedad cardiovascular $^{19-20}$. La PCR promueve además la inflamación de las células endoteliales, estimula la expresión de PAI-1, de moléculas de adhesión vascular, aumenta la síntesis de MCP-1, todos factores que promueven la inflamación endotelial lo que constituye un riesgo para el desarrollo de diabetes y sus complicaciones ${ }^{21}$.

Varios estudios han demostrado la utilidad de metformina en la reducción de los factores de riesgo asociados con diabetes, como el estrés oxidativo, la inflamación endotelial y en la expresión de genes de la inflamación ${ }^{15-16,22}$, sin embargo, hay poca información sobre los efectos de metformina sobre la PCRus. Li et al (2009) ${ }^{23}$ demostraron en un modelo de ateroesclerosis de conejo, que al administrar metformina había una disminución de la PCRus, pero también había disminución de la activación de NF- $\square$ B.- En los sujetos aquí estudiados no encontramos diferencias en la abundancia del RNAm de NF- $\square \mathrm{B}$, sin embargo, el estrés oxidativo está muy ligado a la actividad de este factor nuclear ya que los ROS y los AGEs son inductores de NF- $\square \mathrm{B}^{24}$, en los sujetos diabéticos estudiados no se encontró diferencias ni en la actividad de la enzima hem oxigenasa-1 ni en la concentración de especies reactivas al ácido tiobarbitúrico, ambos marcadores de estrés oxidativo (datos no mostrados). Estos hallazgos sugieren por lo tanto, que los sujetos de ambos grupos tienen un nivel de estrés oxidativo comparable y que podría explicar el hecho que ambos grupos tuvieran una abundancia relativa del RNAm de NF- $\square$ B comparables.

Los pacientes en este estudio además de ser diabéticos eran obesos, el tejido adiposo junto con los macrófagos que lo infiltran son los mayores secretores de IL-6, esta citoquina tiene una fuerte relación con el desarrollo de insulino resistencia y con la inflamación vascular ${ }^{25}$. La expresión de IL-6 en el grupo con tratamiento con metformina no fue distinta a la del grupo sin tratamiento en este estudio, destacando que la expresión de IL-6 está marcadamente elevada en ambos grupos (Grupo metformina vs sin metformina, 98 vs 102 veces).
Otros estudios han mostrado leves reducciones de IL-6 en sujetos tratados con metformina ${ }^{15}$, sin embargo, estos estudios no han considerado individuos obesos, probablemente el aporte del tejido adiposo de IL-6 supera el efecto que metformina pueda tener sobre la represión de esta citoquina, por lo tanto, esto demuestra la importancia del control de la adiposidad en los sujetos con síndrome metabólico y diabetes.

En los sujetos tratados con metformina en este estudio se encontró una menor abundancia relativa de los RNAm de TNF- $\square$ y los TLR $2 / 4$. TNF- $\square$ es una citoquina que cumple un importante rol en el desarrollo de resistencia a la insulina mediante la activación de la vía JNK, lo que promueve la fosforilación en serina de la sub-unidad 1 del receptor de insulina provocando alteración en su señalización ${ }^{26}$. TNF- $\square$ en general se encuentra elevada en sujetos obesos, y esta citoquina cumple la función de amplificar la respuesta inflamatoria ya que promueve la expresión de otras citoquinas pro-inflamatorias, como IL- $6^{6}$, por lo tanto, nuestro hallazgo que metformina participa en la reducción de la expresión de TNF- $\square$ reafirma el carácter anti-inflamatorio de este fármaco.

Asimismo, se encontró una reducción en la expresión de los TLR 2/4, estos receptores participan en la respuesta inmune y recientemente se demostró que además podrían ser activados con glucosa y ácidos grasos. La activación de estos receptores provoca la exacerbación de la inflamación ya que termina en el aumento de expresión de IL-6, IL-1b, TNF- - , MCP-1 etc et12. $^{112}$. Por otra parte, la activación de estos receptores también induce la activación de vías apoptóticas en la célula beta ${ }^{27}$. No existe ninguna evidencia previa que haya demostrado que metformina puede reprimir la activación de los TLR $2 / 4$, por lo tanto, sería interesante conocer más sobre este mecanismo de acción de este fármaco en la inflamación.

En resumen, la metformina es un fármaco demostradamente útil en el manejo de la diabetes, en este estudio observamos que los pacientes que la consumían tenían un menor nivel de PCRus circulante como así mismo menor expresión de TNF- $\square$ y los TLR 2/4, lo que sugiere que la inflamación en los sujetos que consumen metformina está menos exacerbada que en los sujetos que no la consumen. Sin embargo, el tratamiento con metformina no indujo a ningún cambio en el perfil lipídico, en parámetros de estrés oxidativo, ni en la expresión 
de IL-6 y NF- $\square \mathrm{B}$, lo que podría sugerir que los efectos de metformina en estos parámetros se ven influenciado por la adiposidad de los individuos.

\section{Referencias}

1. Cho JH, Kim JW, Shin JA, Shin J, Yoon KH. Beta-cell mass in people with type 2 diabetes. J Diabetes Invest 2011; 2: 6-17.

2. Maedler K, Sergeev P, Ris F, Oberholzer J, Joller-Jemelka HI, Spinas GA, et al. Glucose-induced beta cell production of IL-1beta contributes to glucotoxicity in human pancreatic islets. J Clin Invest 2002; 110: 851-60.

3. Kim JW, Ko SH, Cho JH, Sun C, Hong OK, Lee SH, et al. Loss of beta-cells with fibrotic islet destruction in type 2 diabetes mellitus. Front Biosci 2008; 13: 6022-33.

4. Kaiser N, Leibowitz G, Nesher R. Glucotoxicity and beta-cell failure in type 2 diabetes mellitus. J Pediatr Endocrinol Metab 2003; 16: 5-22.

5. Brownlee M 2000. Negative consequences of glycation. Metabolism 49 (2 Suppl1): 9-13.

6. Hotamisligil GS. Inflammation and metabolic disorders. Nature 2006; 444: 860-86.

7. Shoelson S, Lee J, Goldfine A. Inflammation and insulin resistance. J Clin Invest 2006; 116: 1793-801.

8. Berg AH, Scherer PE. Adipose tissue, inflammation, and cardiovascular disease. Circ Res 2005; 96: 939-49.

9. Gregor M, Hotamisligil G. Inflammatory mechanisms in obesity. Annu Rev Immunol 2010; 29: 415-45.

10. Dandona P, Aljada A, Bandyopadhyay A. Inflammation: the link between insulin resistance, obesity and diabetes. Trends Immunol 2004; 25: 4-9.

11. Dasu M, Devaraj S, Zhao L, Hwang DH, Jialal I. High glucose induces toll-like receptor expression in human monocytes. Diabetes 2008; 57: 3090-8.

12. Dasu M, Jialal I. Free fatty acids in the presence of high glucose amplify monocyte inflammation via Toll-like receptors. Am J Physiol Endocrinol Metab 2010; 300: E145-54.

13. Krentz A, Bailey C. Oral antidiabetic agents. Current role in type 2 diabetes mellitus. Drugs 2005; 65: 385-411.

14. UK Prospective Diabetes Study (UKPDS) Group. Effect of intensive blood-glucose control with metformin on complications in overweight patients with type 2 diabetes (UKPDS 34). Lancet 1998; 352: 854-65.

15. Fidan E, Ersoz HO, Yilmaz M, Yilmaz H, Kocak M, Karahan $\mathrm{C}$, et al. The effects of rosiglitazone and metformin on inflammation and endotelial dysfunction in patients with type 2 diabetes mellitus. Acta Diabetol 2011; 48: 297-302.
16. Chakraborty A, Chowdhury S, Bhattacharyya M. Effect of metformin on oxidase stress, nitrosative stress and inflammatory biomarkers in type 2 diabetes patients. Diab Clin Res. 2011; 93: 56-62.

17. Alhaider A, Korashy H, Sayed-Ahmed M, Mohammed M, Kfoury H, Mansour MA. Metformin attenuates streptozotocin-induced diabetic nephropathy in rats through modulation of oxidative stress genes expression. Chem Biol Interact 2011; 192: 233-42.

18. Libby P, Plutzky J. Diabetic macrovascular disease. The glucose paradox. Circulation 2002; 106: 2760-3.

19. Ridker PM, Buring JE, Cook NR, Rifai N. C-reactive protein, the metabolic syndrome, and risk of incident cardiovascular events: an 8-year follow-up of 14719 initially healthy American women. Circulation 2003; 107: 391-7.

20. Ballantyne CM, Hoogeveen RC, Bang H, et al. Lipoprotein-associated phospholipase A2, highsensitivity $\mathrm{C}$-reactive protein, and risk for incident ischemic stroke in middle-aged men and women in the Atherosclerosis Risk in Communities (ARIC) study. Arch Intern Med 2005; 165: 2479-84.

21. Sjoholm A, Nystrom T. Endothelial inflammation in insulin resistance. Lancet 2005; 365: 610-2.

22. Derosa G, D’Angelo A, Ragonesi PD, Ciccarelli L, Piccini $\mathrm{MN}$, Pricolo F, et al. Metformin-pioglitazone and metformin-rosiglitazone effects on non-conventional cardiovascular risk factors plasma levels in type 2 diabetic patients with metabolic síndrome. J Clin Pharm Ther 2006; 31: 375-83

23. Li SN, Wang X, Zeng QT, Feng YB, Cheng X, Mao XB, et al. Metformin inhibits nuclear factor $\mathrm{kB}$ activation and decreases serum high-sensitivity C-reactive protein level in experimental atherogenesis of rabbits. Heart Vessels 2009; 24: 446-53.

24. Hayden M, Ghosh S. Shared principles in NF- $\square$ B signaling. Cell. 2008; 132: 344-62.

25. Singer G, Granger DN. Inflammatory responses underlying the microvascular dsyfunction associated with obesity and insulin resistance. Microcirculation 2007; 14: 375-87.

26. Plomgaard P, Bouzakri K, Krogh-Madsen R, Mittendorfer B, Zierath J, Pedersen BK. Tumor necrosis factor- $\alpha$ induces skeletal muscle insulin resistance in healthy human subjects via inhibition of Akt substrate 160 phosphorylation. Diabetes 2005; 54: 2939-45.

27. Ehses JA, Meier DT, Wueest S, Rytka J, Boller S, Wielinga PI, et al. Toll-like receptor 2-deficient mice are protected from insulin resistance and beta cell dysfunction induced by a high-fat diet. Diabetologia 2010; 53: 1795-806. 